\title{
Detection and characteristics of magnetic fields in evolved intermediate mass stars
}

\author{
Laurence Sabin
}

Instituto de Astronomía y Meteorología, Departamento de Física, CUCEI, Universidad de Guadalajara, Av. Vallarta 2602, C.P. 44130, Guadalajara, Jal., Mexico email: lsabin@astro.iam.udg.mx

\begin{abstract}
The role of magnetic fields in late type stars, such as Asymptotic Giant Branch stars (AGBs), Post-AGBs and Planetary Nebulae (PNe), is poorly known from an observational point of view. Magnetic fields are however believed to have a non-negligible influence on the dynamics (via mass loss control, outflows shaping) and even on the chemistry (e.g. extra mixing) of these stellar objects. We are therefore presenting two different types of investigation, both based on the use of polarimetry, which aim at filling the gap between the observations on the one hand and the theoretical predictions on the other hand.
\end{abstract}

Keywords. Magnetic fields, polarisation, stars: AGB and post-AGB stars

\section{Introduction}

One of the main issue in the study of magnetic fields in evolved intermediate mass stars is related to their role in the shaping of the nebulae as $80 \%$ of PNe display an aspherical morphology. Other questions related to the field ability to launch and collimate outflows/jets (Blackman et al. 2001), control the mass loss process (García-Segura et al. 2001) and its role in the chemical diffusion from the stellar interior to the atmosphere (Busso et al. 2007) have also yet to be answered. To solve these problems it is necessary to have observational data in order to qualitatively and quantitatively estimate those fields. We therefore need to i) map the magnetic field and infer its distribution at large and small scales and ii) measure the field intensity in order to unveil its characteristics and relationship with other physical parameters (e.g. rotation, mass, mass loss...). Both methods use polarimetric techniques which are applied at different wavelengths.

\section{2. (Sub)-millimeter polarimetry}

We are interested in the detection and study of dust continuum polarisation. The latter is based on the principle of alignment of non-spherical spinning dust grains with their long axis perpendicular to the magnetic field (Lazarian 2003). Thus the linearly polarised emission will provide a map of the field distribution/geometry. In the recent years we used two intereferometric facilities: the Submillimeter (SMA) array (Sabin et al. 2014) and the Combined Array for Research in Millimeter-wave Astronomy (CARMA) (Sabin et al., submitted), to obtain an (inferred) image of the magnetic field in dust-rich evolved stars. One of the most interesting target is the asymmetrical Proto-Planetary Nebula $\mathrm{OH}$ $231.8+4.2$ or Calabash Nebula (see Fig.1-left). The right panel in Fig.1 shows the thermal continuum emission detected with the SMA in blue contours (inner area) over an area of $\sim 7.3 \times 5.7 \operatorname{arcsec}^{2}$ and a mean emission of $0.31 \mathrm{Jy} /$ beam; and the emission detected with CARMA in red contours (outer area) over an area of $\sim 15.3 \times 8.8 \operatorname{arcsec}^{2}$ and a mean emission of $0.076 \mathrm{Jy} /$ beam. 

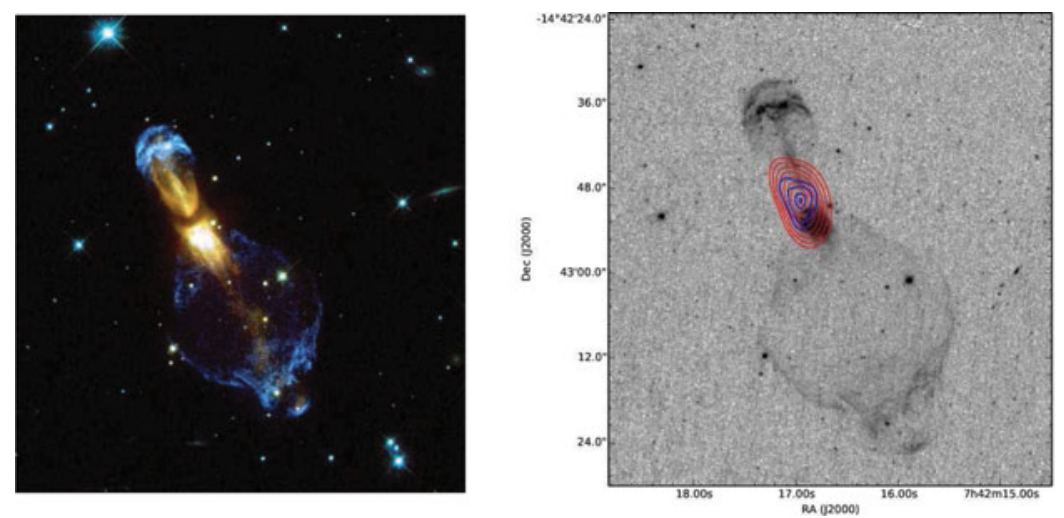

Figure 1. Left: Optical HST image of OH 231.8+4.2. Right: Dust continuum observed with the SMA (inner blue contours at $\sim 345 \mathrm{GHz}$ ), and with CARMA (outer red contours at $\sim 230$ $\mathrm{GHz}$ ) overlaid on the $\mathrm{H} \alpha \mathrm{HST}$ image. See the on-line edition of this book for a color version of this figure.

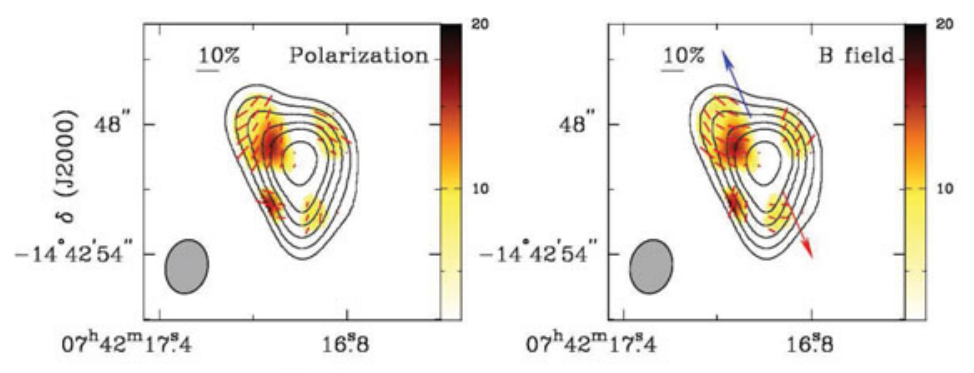

$\alpha(\mathrm{J} 2000)$

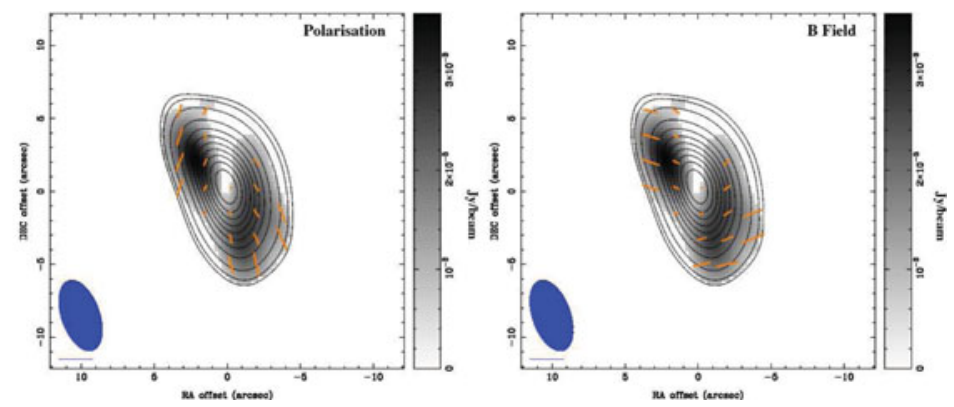

Figure 2. Polarisation (left) and magnetic field (right) maps of $\mathrm{OH} 231.8+4.2$ obtained with the SMA (Top row) and CARMA (Bottom row). In all cases, the black contours indicate the total dust emission, the color or gray scale image indicates the polarised intensity with its associated scale bar in Jy/beam on the right and the polarisation vectors are drawn with a scale set to 10 per cent. Note: on the top panel the arrows indicate the direction of the blue (towards NE) and red-shifted (towards SW) CO molecular emission. See the on-line edition of this book for a color version of this figure.

The polarisation and inferred magnetic field maps from both arrays are presented in Fig.2. In each case linear polarisation was detected above $3-\sigma$ across the source and we measured a mean intensity of $11 \mathrm{mJy} / \mathrm{bm}$ at sub-mm wavelength and $1.7 \mathrm{mJy} / \mathrm{bm}$ at millimeter wavelength. The respective mean fractional polarisation were $4.3 \%$ (SMA) and $3.4 \%$ (CARMA). We note that in those works there was no detection of line polarisation (e.g. in molecules). But the most interesting feature for our purpose is the analysis of 
Table 1. Observing log of the sample presenting for each object the observing date, number of Stokes $V$ sequences, length of the sub-exposures and peak signal-to-noise ratio.

\begin{tabular}{|l|c|c|c|c|c|c|c|}
\hline Parameters & CY CMi & U Mon & V814 Her & 89 Her & AC Her & V4728 Sgr & R Sct \\
\hline Obs date & $2014-02-11$ & $2014-04-10$ & $2009-02-16$ & $2014-06-15 / 19$ & $2014-06-19$ & $2014-06-16$ & $2014-07-21 / 23$ \\
$N^{\circ}$ Stokes V seq. & 2 & 5 & 2 & 8 & 4 & 2 & 6 \\
Subexp.time (s) & 800 & 284 & 600 & 245 & 800 & 800 & 300 \\
Peak S/N & 589 & 1073 & 1028 & 958 & 900 & 885 & $1000-1500$ \\
\hline
\end{tabular}

the magnetic maps i.e. the distribution of the "magnetic vectors". The data therefore indicate i) the presence of a X-shaped structure which can be related to a polar magnetic field configuration, ii) the presence of "magnetic vectors" which follow the CO molecular outflows and iii) the coexistence of a toroidal structure (organised field) at larger scale. These information represent a first step in probing the possible magnetic launching mechanism operating in $\mathrm{OH} 231.8+4.2$. However more data will be needed, as for example, the detection of the magnetic field much closer to the launch-zone of the jets/outflows i.e. at higher resolution with new facilities such as ALMA.

\section{Optical spectropolarimetry}

In addition to the mapping of the magnetic field, an important piece of information is the strength of the latter. We therefore proceeded with the detection of (longitudinal) magnetic fields at the surface of post-AGB stars using the high resolution spectropolarimeters $(\mathrm{R}=65000)$ ESPaDOnS (CFHT, Hawaii) and Narval (TBL, France). Our first sample was composed of seven well known bright objects CY CMi, U Mon, V814 Her, 89 Her, AC Her, V4728 Sgr and R Sct (Sabin et al. 2015). The observing log is presented in Table 1 and we emphasize the high signal-to-noise (above $~ 500$ ) which is needed to unveil any meaningful signal. The data were analysed using the Least Square Deconvolution (LSD) technique (Donati et al. 1997 and Kochukhov et al. 2010) and Fig.3 indicates that among the six targets observed with ESPaDOnS, only U Mon shows a clear Zeeman signature (Stokes $V$ feature). The LSD flag indicates a "definite detection" and corresponds to a longitudinal magnetic field of $10.2 \pm 1.7$ G. The Post-AGB star R Sct was observed with Narval and we also identified a significant but more complex Stokes $V$ signal (more details on this object are in Lèbre et al. 2015). The clear detection in U Mon represents the first clear discovery of weak magnetic fields at the stellar surface of a Post-AGB star. Finally we noted a possible association of the magnetic field with the dynamical state of the atmosphere (seen with the Stokes $I$ double line): the magnetic field would vary with the pulsational phase of the star. Monitoring is therefore needed to prove such hypothesis.

\section{Conclusion}

We present new observational advances in the characterisation of the magnetic field in evolved intermediate mass stars using polarimetric techniques. Thus, the magnetic field mapping via the analysis of the polarisation from dust alignment at large wavelengths (submm/mm) in the envelope, allowed us to define the (multi-scale) field configuration in the PPN OH 1231.8+4.2. This type of data will help us to constrain the capability of the field to launch and collimate the flows. At the stellar surface we obtained the first clear positive detections of photospheric magnetic fields; and these results will open the door to more investigations: for example to unveil the physical parameters linked to 

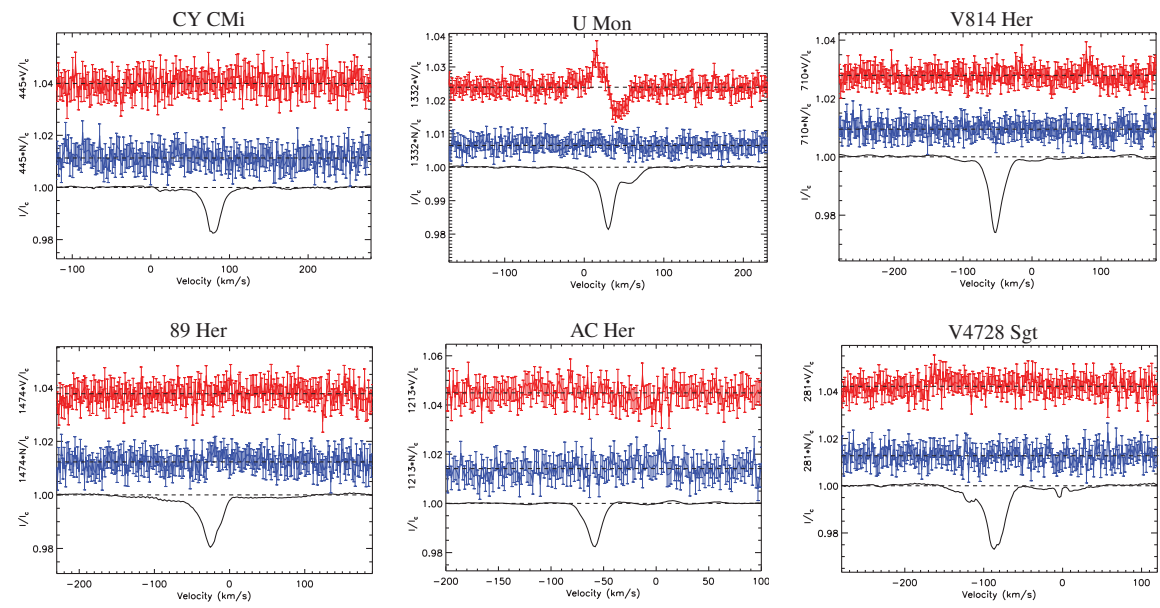

Figure 3. LSD profiles obtained with ESPaDOnS for the post-AGB stars CY CMi, U Mon, V814 Her, 89 Her, AC Her and V4728 Sgr. For each object, the upper plot (in red) shows the mean Stokes $V$ profile, the middle plot (in blue) shows the null $(N)$ profile and the bottom plot (in black) indicates the mean Stokes $I$ profile. See the on-line edition of this book for a color version of this figure.

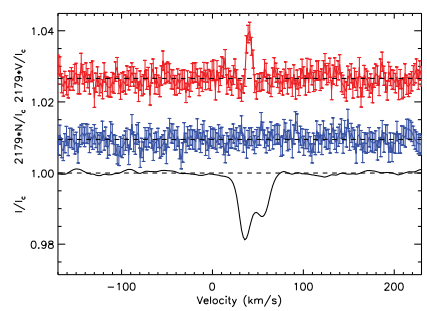

Figure 4. Average LSD profiles obtained with the high resolution spectropolarimeter Narval of R Sct between the 21 and 23-July-2014. We note that the Stokes $V$ profile differs from the classical "S" shape linked to the Zeeman effect. See the on-line edition of this book for a color version of this figure.

the magnetic field as well as its commonality in evolved stars. The monitoring of the "magnetic sources" is needed to investigate the variability of the field and its interaction with the atmosphere dynamics.

All those results and corresponding methods are a first step in the investigation (based on observations) of magnetic fields as mechanisms playing an important role in the structure, dynamics and chemistry of evolved stars.

\section{References}

Blackman, E. G., Frank, A., Markiel, J. A., Thomas, J. H., \& Van Horn, H. M. 2001, Nature 409,485

Busso, M., Wasserburg, G. J., Nollett, K. M., \& Calandra, A. 2007, ApJ 671, 802

Donati, J.-F., Semel, M., Carter, B. D., Rees, D. E., \& Collier Cameron, A. 1997, MNRAS 291, 658

García-Segura, G., López, J. A., \& Franco, J. 2001, ApJ 560, 928

Kochukhov, O., Makaganiuk, V., \& Piskunov, N. 2010, A\&A 524, AA5

Lazarian, A. 2003, JQSRT 79, 881 
Lèbre, A., Aurière, M., Fabas, N., Gillet, D., Josselin, E., Mathias, P., \& Petit, P. 2015, in: K.N. Nagendra, S. Bagnulo, R. Centeno, \& M.J. Martínez González (eds.), Polarimetry: From the Sun to Stars and Stellar Environments, IAU Symp. No. 305, p. 47

Sabin, L., Zhang, Q., Zijlstra, A. A., et al. 2014, MNRAS 438, 1794

Sabin, L., Wade, G. A., \& Lèbre, A. 2015, MNRAS 446, 1988 